\title{
Estimation of Anti Hbs antibody titer in adults during 5-10 years period following three doses of vaccine
}

\author{
Dr.B.S.Mahawal ${ }^{1}$, Dr.N.Bhai ${ }^{2}$, Dr.V.K.Kataria ${ }^{3 *}$, Dr.N.Gulati ${ }^{4}$, Dr.I.Chandola ${ }^{5}$ \\ ${ }^{1,3,5}$ Department of Microbiology, Shri Guru Ram Rai Institute of Medical and Health Sciences, Dehradun \\ ${ }^{2}$ Regency Hospital Kanpur, ${ }^{4}$ LHMC New Delhi \\ *corresponding author
}

\begin{abstract}
Health care professional are a high risk group for Hepatitis $B$ and are advised vaccination against hepatitis $B$. The protective antibodies induced by Hepatitis $B$ vaccination wane gradually over period of time and may reach very low or even undetectable levels. A cross sectional prospective study was undertaken to investigate the persistence of anti-HBs levels in health care professionals who had been immunized with $H B V$ vaccine and the need for booster doses of the vaccine. In this cross-sectional study, health care professionals (18 -55 yrs of age) who had received full course of HBV vaccination were sampled and tested for anti-HBs from May 2010 to June 2011. Plasma samples were tested for anti-HBs using ELISA. Titer $>=10 \mathrm{mIU} / \mathrm{mL}$ was considered to be seroprotective. Individuals with titre $<10 \mathrm{mIU} / \mathrm{mL}$ were advised full three doses of vaccine. A total of 112 samples were collected. Protective antibody levels were detected in $99.9 \%$ of subjects one year after vaccination. It decreased to $80.96 \%$ by 5 years after vaccination, and further decreased to $46.16 \%$ in 10 years after vaccination. Sero protection rates decreased significantly with increasing time from last vaccination due to waning anti-HBs titer $(P<0.001)$.According to the above mentioned results, for a high risk group population such as medical students and residents, who are at continuous exposure to $H B V$, it is reasonable to determine the anti HBsAb response at one month post vaccination However, in order to confirm the persistence of immune protection, we strongly suggest detection of the anti-HBsAb titer at 5 - 10 years after the last inoculation.
\end{abstract}

Key words: Anti HBs, ELISA, vaccination, seroprotection rates

\section{Introduction}

Hepatitis B virus is highly infectious and causes serious health problems worldwide. Approximately one third of the world's population has been infected, and 400 million have become chronic carriers The infection results in a wide spectrum of diseases, ranging from hyperacute fulminant hepatitis to refractory cirrhosis and hepatocellular carcinoma. Infections that occur at a younger age frequently result in chronic carriage of the virus. In contrast, only a small proportion of patients become chronic carriers if they contact the infection in adolescence or adulthood. Once the infection becomes chronic, HBV or part of the viral genome persists in the liver for the carrier's life time. The carriers are not only reservoirs of the virus but also victims of chronic liver diseases themselves. Thus control of HBV infection especially in terms of preventing chronic carriage of the virus, is extremely important ${ }^{1}$.

Hepatitis B vaccine was first licensed for use in Canada in 1982.Initial follow up studies indicated that $5 \mathrm{yrs}$ after the primary course of vaccination a significant proportion of the recipients had a level of antibody (anti-Hbs) to hepatitis B surface antigen ( $\mathrm{HBsAg}$ ) below that believed to be required for protection $(10 \mathrm{mIU} / \mathrm{ml})$. On this basis the National Advisory Committee on Immunization (NACI) recommended that a booster be given 5 yrs after completion of the primary series but acknowledged that the need for and timing of booster doses were not known with certainty ${ }^{2}$. Thereafter many studies were carried out throughout the world regarding this with varied results.

Health care professional are a high risk group for Hepatitis B and are advised vaccination against hepatitis B. Though studies from Western countries have shown strong and rapid response to boosters in adult ${ }^{3}$, there is little data from India on this aspect. Vaccinated individuals having an anti-Hbs level $10 \mathrm{mIU} / \mathrm{ml}$ are generally considered protective. However, the protective antibodies induced by Hepatitis B vaccination wane gradually over time and may reach very low or even undetectable levels ${ }^{4}$. The real duration of protection conferred by HBV vaccination remained uncertain. The necessity of booster vaccination for HBV has been a subject of much debate.

A lot of conflicting ideas exist regarding post vaccination antibody titers. ${ }^{4,5,11}$. Hence a cross sectional prospective study was undertaken to investigate the persistence of anti-HBs levels in health care professionals who had been immunized with $\mathrm{HBV}$ vaccine and the need for booster doses of the vaccine.

\section{Aims and Objective}

- $\quad$ Estimate the anti HBs antibody titer in adults during 10 yrs period post vaccination 


\section{Material and Methods}

In this cross-sectional study, health care professionals (18 -55 yrs of age) who had received full course of HBV vaccination were sampled and tested for anti-HBs from May 2010 to June 2011

Those suffering from chronic diseases or having received intravenous immunoglobulin (IVIG) were excluded from the study. institute,

Informed consent was obtained from all. The study was approved by the ethics committee of the

Plasma was obtained after centrifugation from the samples and stored at $-80^{\circ} \mathrm{C}$ prior to testing. Plasma samples were tested for anti-HBs using enzyme-linked immunosorbent assay (ELISA) ANTI-HBs IEMA WELL RADIM kit following the manufacturer's protocol.

The levels of anti-HBs titer were grouped as follows:

$\begin{array}{ll}\text { 1. } & <10 \mathrm{mIU} / \mathrm{mL} \\ 2 . & 10-100 \mathrm{mIU} / \mathrm{mL} \\ \text { 3. } & 100-1000 \mathrm{mIU} / \mathrm{ml} \\ 4 . & >1000 \mathrm{mIU} / \mathrm{mL}\end{array}$

Titer $>=10 \mathrm{mIU} / \mathrm{mL}$ was considered to be seroprotective. Individuals with titer $<10 \mathrm{mIU} / \mathrm{mL}$ were advised full three doses of vaccine.

The Chi-square test and Fisher's exact test were used with the SPSS 16 Package program (Chicago, IL, USA). Data were presented as an absolute number and percentage.

\section{Results}

A total of 112 samples were collected .Of these 7 were hemolyzed. No repeat samples were obtained for these.

Of the 105 individuals studied $45(40.17 \%)$ were females and 67(59.82\%) were males

Subjects were divided into three groups based on the time from last dose of vaccine

Group A 1-4 years post vaccination

Group B 5-9 years post vaccination

Group C >10 yrs Post vaccination

The serological profiles of anti HBs of the three study groups mentioned above are as detailed in Table 1

Protective antibody levels were detected in $99.9 \%$ of subjects one year after vaccination, decreased to 80.96 by 5 years after vaccination, and further decreased to $46.16 \%$ in 10 years after vaccination, respectively. Sero protection rates decreased significantly with increasing time from last vaccination due to waning anti-HBs titer over time $(\mathrm{P}<0.001)$

\section{Discussion}

HBV remains a worldwide major pathogenic factor for chronic liver disease. In order to protect the health care personnel including the medical students and residents (as the future physicians), it is necessary to be certain about their immune response in vaccination era. In this study, almost all (99.9\%) of vaccinees had protective level of antibody ( $>10 \mathrm{mIU} / \mathrm{mL})$ at the end of one year. It declined to $80.96 \%$ in5-9 year interval and to $46.16 \%>10$ years after inoculation. There was no immune non responder in our study.

There is still uncertainty about the persistence of the vaccine induced protection and the need for revaccination among different authors. ${ }^{4,5,11}$. One opinion is that the vaccine length of protection is related to the maximum level of anti-HBs response. The maximum anti-HBs titers is reached between 1 and 2 months after the last vaccine dose, then it begins to decrease rapidly during $20-24$ months, but subsequently the fall is slower ${ }^{5}$. In Dienstag study on 658 health care personnel, levels of anti-HBsAb increased gradually during the months after plasmatic HBV vaccination. It was positive in $96.6 \%$ of vaccine recipients at 12 months and in $98.9 \%$ at 18 months, when the study ended ${ }^{6}$. Chadha ${ }^{7}$ studied the long-term persistence of anti-HBsAb in 34 health care workers following three dose injections. In that study, group $\mathrm{A}(\mathrm{n}=16)$ received booster vaccine after 3 years whereas group B $(n=18)$ did not. After 10 years of follow up, 6/15 $(40 \%)$ of group A and 3/16 $(\sim 19 \%)$ of group B had protective anti-HBsAb. They concluded that persistence of immunological memory remains for at least 10 years. We believe that the comparison of antibody titer in these three groups with different vaccination time interval can replace the long-term follow-up of one single group over time. According to the results of the present study, there was a reduction in Ab titer during years after vaccination, but $84 \%$ of vaccinees had protective level of $\mathrm{Ab}$ in their sera even for more than 5 years. The mean of Ab titer after 5 years was

$250 \pm 59.3 \mathrm{mIU} / \mathrm{mL}$. This implies maintenance of immunological memory for at least $5-10$ years. The results obtained in this study are in agreement with the results of other studies done in India ${ }^{2,4}$ and also in other countries $^{3,8}$ 


\section{Conclusion}

According to the above mentioned results, for a high risk group population such as medical students and residents, who are at continuous exposure to $\mathrm{HBV}$, it is reasonable to determine the anti HBsAb response at one month post vaccination. However, in order to confirm the persistence of immune protection, we strongly suggest detection of the anti-HBsAb titer at $5-10$ years after the last inoculation. So, the revaccination schedule could be arranged on the basis of personal need without incurring the cost of revaccination for the whole target population.

\section{Acknowledgements}

We would like to express our sincere thanks to Mrs. Vijaylakshmi, senior technician at central lab of our Hospital for all the work done during the course of study

\section{References}

[1]. Acharya SK, Panda SK, Duphare H. Chronic Hepatitis in a large Indian Hospital. Natl Med J India 1993; 6: 202-6.

[2]. Aggarwal R, Naik SR. Prevention of Hepatitis B Infection: The Appropriate Strategy for India. Natl Med J India 1994; 7: 216220 .

[3]. Garuz R, Torrea JL, Arnal JM, Forcen T, Trinxet C, Anton F, Antonanzas F. Vaccination against Hepatitis B virus in Spain: A Cost-Effectiveness Analysis. Vaccine 1997; 15: 1652-60

[4]. Hepatitis B virus: A Comprehensive Strategy for Eliminating Transmission in the United States through Universal Childhood Vaccination. Recommendations of the Immunization Practices Advisory Committee (ACIP). MMWR Recomm Rep 1991; 40:1 25. 4

[5]. West DJ, Calandra GB. Vaccine Induced Immunologic Memory for Hepatitis B Surface Antigen: Implications for Policy on Booster Vaccination. Vaccine 1996; 14:1019-27. ^

[6]. Kant L, Hall AJ. Epidemiology Of Childhood Hepatitis B In India: Vaccination Related Issues. Indian J Pediatr 1995; 62: 635-53.

[7]. Hatziandreu EJ, Hatzakis A, Hatziyannis S, Kane MA, Weinstein MC. Cost effectiveness of Hepatitis-B Vaccine In Greece. A Country of Intermediate HBV Endemicity. Int J Technol Assess Health Care 1991; 7: 379-402.

[8] Jules L. Dienstag, M.D. Hepatitis B Virus Infection. N Engl J Med 2008; 359:1486-1500.

[9] Gandhi S.S, Chadha MS, Arankalle VA. Hepatitis B Virus Genotypes And Serotypes In Western India: Lack Of Clinical Significance. Hepatitis Division, National Institute of Virology, Pune.J Med Virol. 2003 Mar; 69(3):324-30.

[10]. Demicheli V, Jefferson TO. Cost-Benefit Analysis of The Introduction of Mass Vaccination Against Hepatitis B In Italy. J Public Health Med 1992; 14: 367-75.

[11]. Yoshida T, Saito I. Hepatitis B Booster Vaccination For Healthcare Workers. Lancet. 2000 Apr 22; 355(9213):1464

Table 1: Serologic profiles of anti-HBs of three study Groups Group A (1-4 years post vaccination)

Total subjects: 35

\begin{tabular}{|l|l|l|}
\hline Level of antibody titre & Number of subjects & Percentage of subjects $(\%)$ \\
\hline$>1000 \mathrm{mIU} / \mathrm{ml}$ & 6 & 16.00 \\
\hline $100-1000 \mathrm{mIU} / \mathrm{ml}$ & 22 & 64.00 \\
\hline $10-100 \mathrm{mIU} / \mathrm{ml}$ & 7 & 20.00 \\
\hline$<10 \mathrm{mIU} / \mathrm{ml}$ & 0 & 0.00 \\
\hline
\end{tabular}

Group B (5-9 years post vaccination)

Total subjects: 25

\begin{tabular}{|l|l|l|}
\hline Level of antibody titre & Number of subjects & Percentage of subjects $(\%)$ \\
\hline$>1000 \mathrm{mIU} / \mathrm{ml}$ & 4 & 16.0 \\
\hline $100-1000 \mathrm{mIU} / \mathrm{ml}$ & 9 & 36.0 \\
\hline $10-100 \mathrm{mIU} / \mathrm{ml}$ & 7 & 28.0 \\
\hline$<10 \mathrm{mIU} / \mathrm{ml}$ & 5 & 20 \\
\hline
\end{tabular}

Group C (>10 years post vaccination) Total subjects: 45

\begin{tabular}{|l|l|l|}
\hline Level of antibody titre & Number of subjects & Percentage of subjects $(\%)$ \\
\hline$>1000 \mathrm{mIU} / \mathrm{ml}$ & 3 & 7.69 \\
\hline $100-1000 \mathrm{mIU} / \mathrm{ml}$ & 6 & 12.86 \\
\hline $10-100 \mathrm{mIU} / \mathrm{ml}$ & 12 & 25.61 \\
\hline$<10 \mathrm{mIU} / \mathrm{ml}$ & 24 & 53.84 \\
\hline
\end{tabular}


Graph 1. Serologic profiles and geometric mean titers of anti-HBs of three study Groups Serologic profiles of the three study Groups

80

60

40

20

0

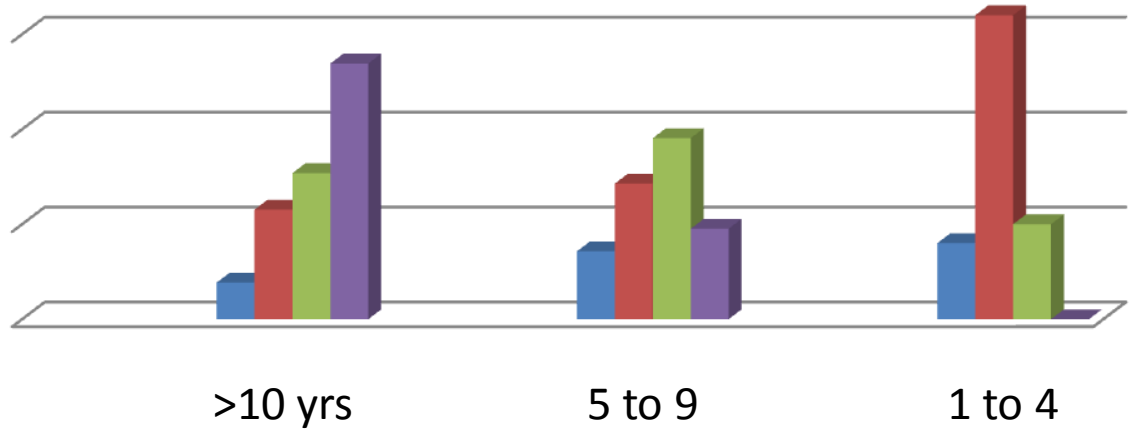

$>1000 \square$ 100-1000 $\quad 10-100 \square<10$ 